\title{
Modification de la technique des zymogrammes en milieu gélosé, en vue du diagnostic rapide des Candida
}

\author{
par M. QUILICI \\ Laboratoires de Parasitologie du C.H.U. et de la Faculté de Médecine de Marseille \\ (Professeurs: J. SAUtet et J. RANQUE)
}

\begin{abstract}
Résumé
Dans la technique des zymogrammes en milieu gélosé, l'adjonction d'un bouchon de paraffine coulée en surface après l'ensemencement permet une lecture plus aisée et plus rapide.
\end{abstract}

\section{Summary}

With the technic of zymograms in agar-medium, we use to adjoin a paraffin stopper laying on the surface after plating. Thus we get rapidly an easier reading. 
Marcelou-Kini et Vournous M., ont décrit une nouvelle 'méthode d'étude du pouvoir fermentatif des Candida.

Le milieu liquide habituellement employé dans les diverses autres techniques est remplacé par un milieu solide.

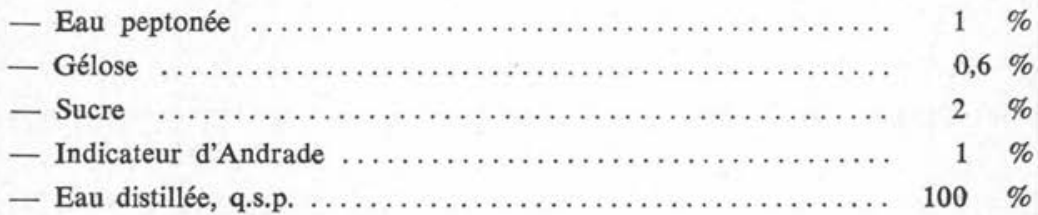

Nous avons appliqué cette technique en routine quotidienne de laboratoire parallèlement à la technique en tubes d'Yvan-Hall.

Nous avons constaté que si l'acidification était rapide dans les tubes gélosés, la production de gaz n'était pas toujours aussi nette.

Lorque la fermentation n'était pas très brutale, les bulles s'éliminaient en cheminant à travers la gélose sans la fragmenter et, si l'on n'examinait pas très souvent les tubes durant les 48 premières heures, cette fermentation pouvait passer inaperçue.

Nous avons essayé d'augmenter la teneur en gélose du milieu mais sans obtenir de résultats plus probants.

Nous avons alors adapté à cette technique l'artifice imaginé par Guerra en 1935, consistant à couler un bouchon de paraffine de 2 à $3 \mathrm{~mm}$ d'épaisseur à la surface de la gélose.

On ensemence en profondeur en piquant jusqu'au fond du tube une öse assez fortement chargée de levures. Ce n'est qu'après l'ensemencement que l'on coule la paraffine.

En cas de fermentation le bouchon est soulevé, parfois jusqu'au niveau du coton obstruant le tube. Il ne faut pas tenir compte des petites bulles qui se forment sous la couche de paraffine. Si la réaction est positive, celle-ci doit être nettement séparée de la gélose.

Depuis plus d'un an nous utilisons cette méthode de façon habituelle, parallèlement à la technique des tubes d'Yvan-Hall. Les résultats ont toujours été nets, concordants et surtout beaucoup plus rapides.

\section{Bibliographie}

Drouhet (E.), 1957. - Biologie des infections à Candida. Diagnostic de Laboratoire. Sem. Hôp. Paris, 33, 793-806.

Guerra (P.), 1935. - Thèse Médecine, Paris. 


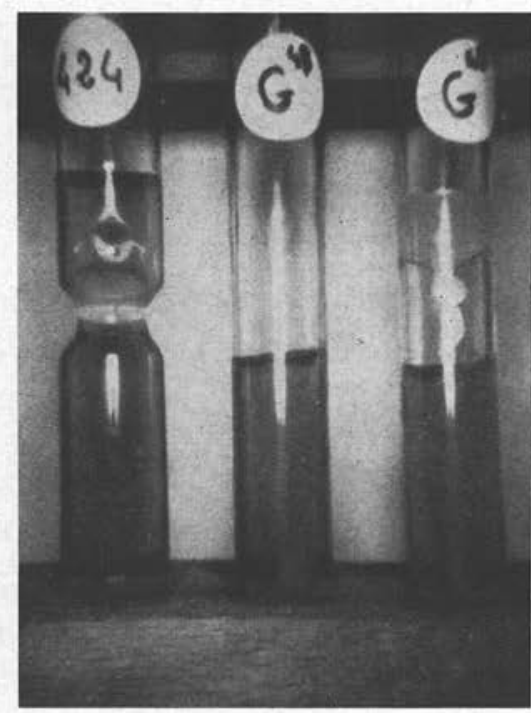

Fig. 1. - Comparaison de la mise en évidence du pouvoir fermentatif d'une même souche, 48 heures après ensemencement. De gauche à droite : en tube de Yvan Hall; en milieu gélosé ; en milieu gélosé + paraffine

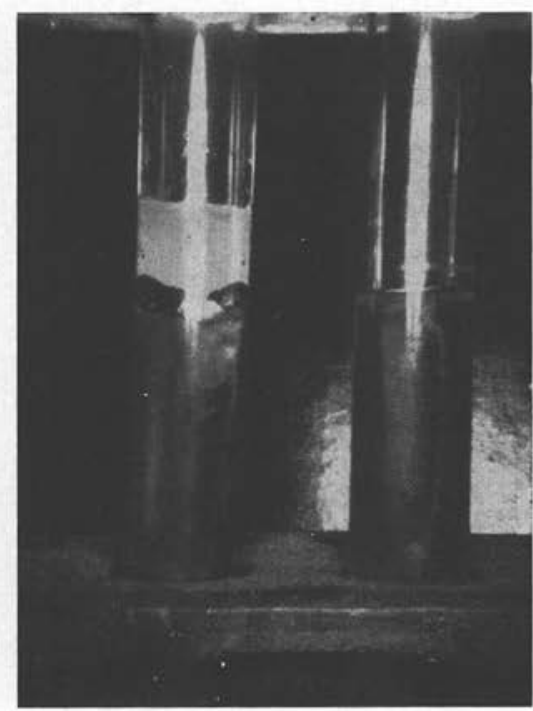

FIG. 2. - Exemple de fermentation passant inaperçue dans le tube gélosé sans bouchon de paraffine.

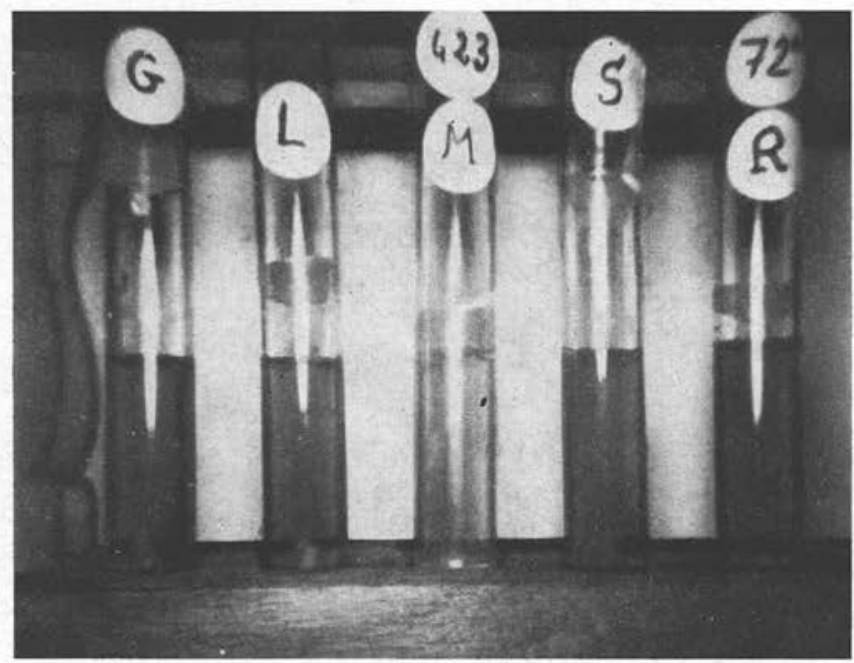

FIG. 3. - Galerie de Candida pseudo-tropicalis en milieu gélosé + paraffine, 72 heures après l'ensemencement. 
Langeron (M.) et Guerra (P.), 1938. - Nouvelles recherches de Zymologie médicales, Ann. Parasit. Hum. Comp., 16-36.

LODDER (J.), 1934. - Die Curaskosporogen Hefen, 1 Halfte Verhandel Kronninkl. Akad Wetenschap. Aft Naturkund. Sect. II, 32, 1.

- et Kréger van RiJ (N.-J.-W.), 1952. - The yeasts, Amsterdam.

Marcélou-Kinti (U.) et Vournous (M.), 1961. - Méthode simple pour l'étude du pouvoir fermentatif des Candida. Cercle Institut Pasteur, Hellen, 7, 163-166. 